\title{
Levantamento Fitossociológico em Pastagens de Várzea no Estado do Amazonas ${ }^{1}$
}

\author{
Phytosociological Assessment of Pastures under Flooded Low Land Conditions in the State of \\ Amazon, Brazil
}

GALVÃO, A.K.L. ${ }^{2}$, SILVA, J.F. ${ }^{3}$, ALBERTINO, S.M.F. ${ }^{4}$, MONTEIRO, G.F.P. ${ }^{5}$ e CAVALCANTE, D.P. ${ }^{5}$

\begin{abstract}
RESUMO - O levantamento fitossociológico em pastagens é uma importante ferramenta de suporte às recomendações de manejo. Objetivou-se com este trabalho realizar um levantamento fitossociológico em pastagens de várzea do município de Autazes-AM. O estudo foi realizado em duas áreas de várzea sazonalmente inundadas, localizadas nesse município. No estudo, utilizou-se o método do quadrado, aplicado por meio de um quadrado de $1,0 \mathrm{~m}^{2}$, lançado ao acaso 31 vezes em cada área de três hectares. Nas duas áreas foram encontrados 27.706 individuos, distribuídos em 12 famílias e 22 espécies. As familias mais importantes em número de espécies nas duas áreas foram Poaceae (7) e Cyperaceae (4). Brachiaria subquadripara mostrou, nas duas áreas, maiores valores de frequência, densidade, abundância e IVI. As espécies que apresentaram maiores valores de IVI na área 1 foram: B. subquadripara $(118,45)$, Paspalum fasciculatum $(51,25)$, Cynodon dactylon $(46,99)$ e Lindernia crustacea $(25,25)$, e na área 2 foram: B. subquadripara $(111,66)$, C. dactylon $(55,44)$, Acroceras zizanioides $(33,70)$ e L. crustacea $(24,91)$. Em ambas as áreas, a familia Poaceae representou mais de $90 \%$ do total de individuos encontrados, e algumas espécies apresentaram bom potencial forrageiro.
\end{abstract}

Palavras-chave: fitossociologia, pastagem, pecuária, plantas daninhas.

\begin{abstract}
The phytosociological assessment of pastures is an important support tool for management recommendations. The objective of this work was to make a phytosociological assessment of flooded low land in Autazes-Amazon, Brazil. The study was carried out in two areas that had been flooded in the past. The inventory square method was applied using a square frame of $1.0 \mathrm{~m}^{2}$, randomly thrown 31 times in each area of three hectares. In these two areas, 27, 706 individuals were found, distributed in 12 families and 22 species. The most important families in number of species in the two areas were Poaceae (7) and Cyperaceae (4). Brachiaria subquadripara presented higher frequency, density, abundance and IVI values in both areas. The species $\boldsymbol{B}$. subquadripara (118.45), Paspalum fasciculatum (51.25), Cynodon dactylon (46.99) and Lindernia crustacea (25.25) presented the highest IVI values in area 1 and the species B. subquadripara (111.66), C. dactylon (55.44), Acroceras zizanioides (33.70) and L. crustacea (24.91) the highest values in area 2. In both areas, the Poaceae family accounted for more than $90 \%$ of the total of the individuals found, and some species presented good forage potential.
\end{abstract}

Keywords: phytosociology, cattle, pasture, weeds.

1 Recebido para publicação em 6.3.2010 e na forma revisada em 18.2.2011.

Parte da tese de doutorado em Agronomia Tropical na FCA/UFAM, da primeira autora. Projeto financiado pela FAPEAM.

2 Doutoranda do Programa de Pós-Graduação em Agronomia Tropical, Faculdade de Ciências Agrárias, Universidade Federal do Amazonas - FCA/UFAM, bolsista CAPES/FAPEAM, Professora de Zootecnia, IFAM/CMZL, <aklg2@yahoo.com.br>, Av. General Rodrigo Octávio Jordão Ramos, 3000, Campus Universitário, Coroado I - Setor Sul, Bloco A, Coordenação do PPG-AT, 69077-000, Manaus-AM; ${ }^{3}$ Professor Associado, Dr., Dep. de Produção Animal e Vegetal - DPAV/UFAM, <jfsilva@ufam.edu.br>; ${ }^{4}$ Doutoranda do Programa de Pós-Graduação em Agronomia Tropical, FCA/UFAM, bolsista CAPES/FAPEAM, <sonialbertino@ufam.edu.br>; ${ }^{5}$ Mestrandos do Programa de Pós-Graduação em Agronomia Tropical, FCA/UFAM, Bolsistas CNPq. 


\section{INTRODUÇÃO}

As pastagens representam a base da alimentação do rebanho bovino no Brasil (Costa et al., 2008), que, em 2008, somou 202,2 milhões de cabeças (IBGE, 2010). No entanto, os índices de produtividade do rebanho, na maioria das propriedades, são considerados insatisfatórios. Alguns fatores que contribuem para isso são a baixa produtividade e a qualidade da forragem; a inexistência de práticas de manejo; a degradação de grandes áreas de pastagens; e animais de baixo potencial produtivo (Costa et al., 2008).

A infestação por plantas daninhas é um dos problemas resultantes da degradação, causada pelo manejo inadequado das pastagens, que, devido à sua capacidade de interferência, reduz a produtividade das forrageiras. Ao competir pelos fatores de crescimento, as plantas daninhas promovem queda da capacidade de suporte da pastagem, aumentam o tempo de formação e de recuperação do pasto, podem causar ferimentos e/ou intoxicação aos animais e comprometem a estética da propriedade (Tuffi Santos et al., 2004).

O levantamento fitossociológico é importante na obtenção do conhecimento sobre as populações e a biologia das espécies encontradas, constituindo importante ferramenta de suporte técnico nas recomendações de manejo e tratos culturais, seja na implantação, recuperação ou condução das pastagens (Mascarenhas et al., 2009).

Apesar de existirem vários trabalhos que estudaram a composição de plantas daninhas em pastagens em diversos Estados (Peixoto et al., 1982; Modesto Júnior \& Mascarenhas, 2001; Lara et al., 2003; Maciel et al., 2008; Tuffi Santos et al., 2004; Mascarenhas et al., 2009), no Amazonas os trabalhos se restringem basicamente a florestas de terra firme e áreas agrícolas (Souza et al., 2003; Albertino et al., 2004; Oliveira \& Amaral, 2004). Nesse Estado, a pecuária sempre se caracterizou por criações em pastagens de várzea (Ohly \& Hund, 1996).

Nesse sentido, o objetivo deste trabalho foi realizar um levantamento fitossociológico em pastagens de várzea do município de Autazes, Estado do Amazonas.

\section{MATERIAL E MÉTODOS}

O levantamento foi realizado em duas áreas de várzea sazonalmente inundadas, localizadas no município de Autazes-AM (Figura 1). As áreas de pastagens estudadas foram: área 1 (S 03 36' 01.7" e W 059 08' 05.7") e área 2 (S 03 $34^{\prime} 47.3^{\prime \prime}$ e W 059 08' 34.2"), de
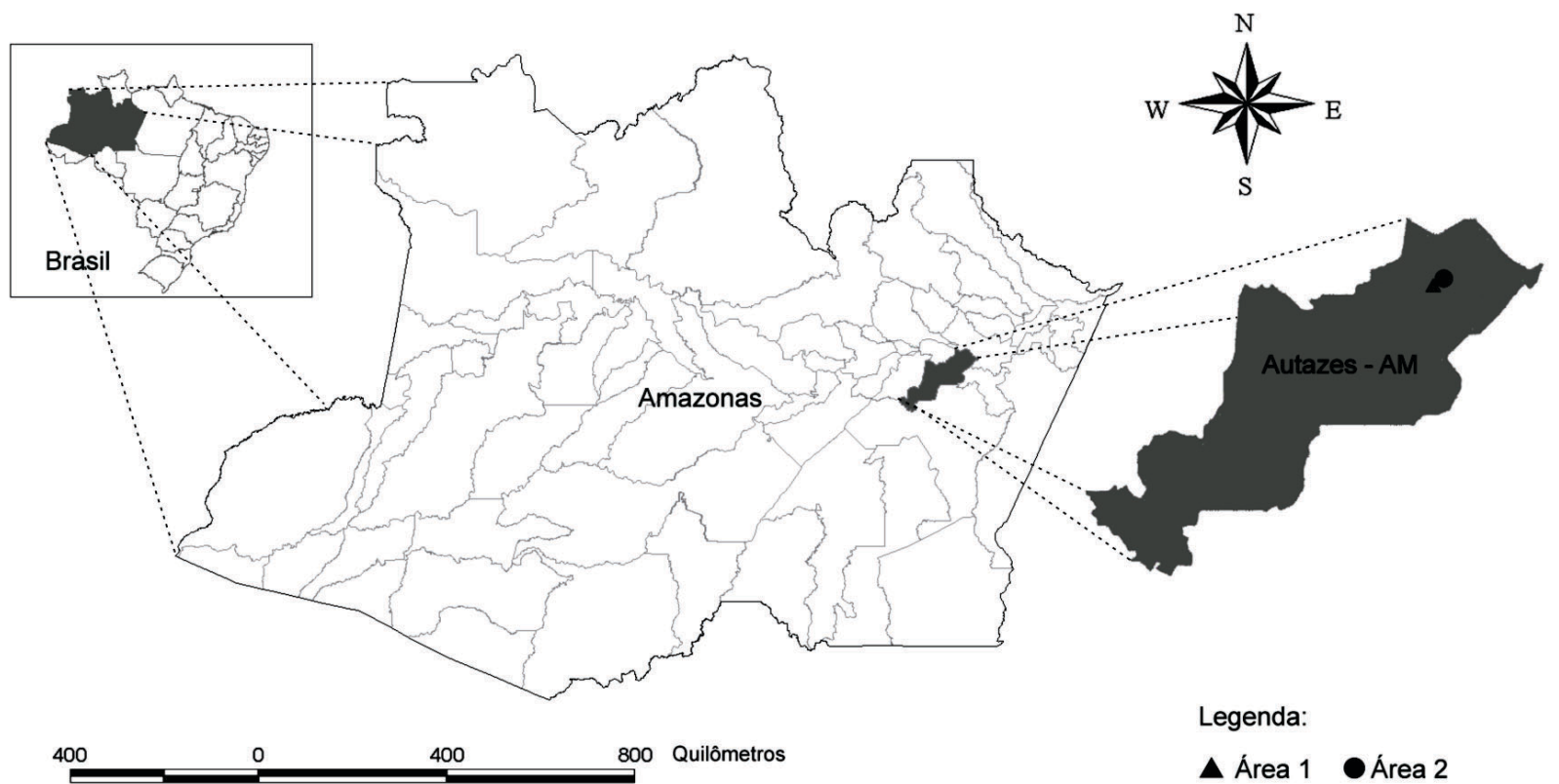

Legenda:

A Área 1 Área 2

Figura 1 - Localização de duas áreas de pastagens de várzea, em Autazes-AM. 
cerca de três hectares cada, sem estratégia de manejo definida e com ausência de adubação.

O levantamento foi realizado no mês de janeiro de 2009, quando as pastagens encontravam-se sob pastejo por bovinos. No levantamento fitossociológico foi usado o método do quadrado, aplicado por meio de um quadrado de madeira medindo $1 \mathrm{~m}$ de lado, o qual foi lançado 31 vezes em cada área estudada, totalizando uma área amostral de $31 \mathrm{~m}^{2}$. As parcelas foram amostradas ao acaso e os terrenos percorridos em ziguezague.

As plantas em cada área amostrada foram cortadas rente ao solo, acondicionadas em sacos plásticos identificados e conduzidas para o laboratório, onde foram quantificadas. Para a identificação das espécies, foram coletados ramos ou fragmentos desses indivíduos, preferencialmente férteis, para que fosse feita sua identificação posterior.

Amostras das plantas foram armazenadas em jornais e prensas, para posterior secagem e confecção de exsicatas. As plantas foram identificadas por classe, família e espécie, por meio de literatura especializada e comparações com material de herbário, e, quando necessário, remetidas a especialistas. Os nomes botânicos apresentados neste estudo foram conferidos com a página da Web do Missouri Botanical Garden (http: / / www.tropicos.org).

Os parâmetros fitossociológicos avaliados foram: frequência $(\mathrm{Fre})$ = número de parcelas que contêm a espécie/ número total de parcelas utilizadas; densidade $($ Den $)=$ número total de indivíduos por espécie/área total coletada; abundância $(\mathrm{Abu})=$ número total de indivíduos por espécie/número total de parcelas que contêm a espécie; frequência relativa $($ Frr $)=$ frequência da espécie $\times 100 /$ frequência total de todas as espécies; densidade relativa $($ Der $)=$ densidade da espécie $\times 100 /$ densidade total de todas as espécies; abundância relativa $(\mathrm{Abr})=$ abundância da espécie x 100/abundância total de todas as espécies; e indice de valor de importância $(\mathrm{IVI})=$ Frr + Der + Abr $($ MuellerDombois \& Ellenberg, 1974).

$\mathrm{Na}$ análise da similaridade florística entre as áreas foi usado o diagrama de Venn, além dos indices de similaridade de Jaccard (Sj) e de Sorensen (IS) (Mueller-Dombois \& Ellenberg,
1974). Os dois indices foram usados com o objetivo de verificar se a diferença existente na sua formulação interfere significativamente na interpretação dos resultados.

As fórmulas usadas no cálculo de similaridade foram: $\mathrm{Sj}=[(\mathrm{a} / \mathrm{a}+\mathrm{b}+\mathrm{c}) \times 100]$ e IS $=$ [(2c/2c+a+b) x 100], em que $a=$ número de espécies exclusivas da área $\mathrm{a} ; b=$ número de espécies exclusivas da área $\mathrm{b}$ e $c=$ número de espécies comuns às duas áreas (MuellerDombois \& Ellenberg, 1974).

\section{RESULTADOS E DISCUSSÃO}

Na área 1 foram identificadas 17 espécies, pertencentes a 10 famílias botânicas (Tabela 1); e na área 2, foram identificadas 14 espécies, pertencentes a oito famílias (Tabela 2).

As famílias mais importantes em número de espécies nas duas áreas foram Poaceae (7) e Cyperaceae (4). Esses resultados estão em concordância com os encontrados por Albertino et al. (2009) em estudo fitossociológico em área de várzea do Estado do Amazonas.

Nas duas áreas foram encontrados 27.706 indivíduos, distribuídos em 12 famílias e 22 espécies. Das espécies identificadas, 13 são monocotiledôneas e nove dicotiledôneas. Em ambas as áreas, a família Poaceae representou mais de $90 \%$ do total de indivíduos encontrados. Brachiaria subquadripara foi a espécie que mais se destacou, apresentando, nas duas áreas, maiores valores de frequência, densidade, abundância e IVI (Tabelas 1 e 2).

As espécies com os maiores valores de IVI na área 1 foram: B. subquadripara $(118,45)$, Paspalum fasciculatum $(51,25)$, Cynodon dactylon $(46,99)$ e Lindernia crustacea $(25,25)$; e na área 2: B. subquadripara $(111,66)$, C. dactylon $(55,44)$, Acroceras zizanioides $(33,70)$ e $L$. crustacea $(24,91)$.

B. subquadripara está presente em vários ecossistemas de água doce no Brasil (Carvalho et al., 2003; Martins et al., 2008; Thomaz et al., 2009). É muito comum o uso do nome Brachiaria arrecta para essa espécie, mas, segundo Kissmann \& Groth (1997), o correto é B. subquadripara. A espécie é originária da África e foi introduzida no Brasil como planta forrageira, escapando logo em seguida para 
Tabela 1 - Famílias e espécies de plantas encontradas na área 1 e respectivos parâmetros fitossociológicos

\begin{tabular}{|c|c|c|c|c|c|c|c|c|c|}
\hline Espécie & NTI & NPP & FRE & DEN & $\mathrm{ABU}$ & FRR & DER & $\mathrm{ABR}$ & IVI \\
\hline \multicolumn{10}{|c|}{ Amarantaceae } \\
\hline Alternanthera philoxeroides (Mart.) Griseb & 99 & 3 & 0,10 & 3,19 & 33,00 & 2,05 & 0,64 & 3,35 & 6,05 \\
\hline \multicolumn{10}{|c|}{ Arecaceae } \\
\hline Astrocaryum gynacanthum Mart. & 1 & 1 & 0,03 & 0,03 & 1,00 & 0,68 & 0,01 & 0,10 & 0,79 \\
\hline \multicolumn{10}{|c|}{ Cyperaceae } \\
\hline Cyperus sphacelatus Rottb. & 75 & 10 & 0,32 & 2,42 & 7,50 & 6,85 & 0,49 & 0,76 & 8,10 \\
\hline Fimbristylis miliacea (L.) Vahl & 71 & 7 & 0,23 & 2,29 & 10,14 & 4,79 & 0,46 & 1,03 & 6,29 \\
\hline \multicolumn{10}{|c|}{ Euphorbiaceae } \\
\hline Croton glandulosus L. & 1 & 1 & 0,03 & 0,03 & 1,00 & 0,68 & 0,01 & 0,10 & 0,79 \\
\hline \multicolumn{10}{|c|}{ Fabaceae - Faboideae } \\
\hline Aeschynomene fluitans Peter & 5 & 2 & 0,06 & 0,16 & 2,50 & 1,37 & 0,03 & 0,25 & 1,66 \\
\hline Desmodium barbatum (L.) Benth. & 125 & 6 & 0,19 & 4,03 & 20,83 & 4,11 & 0,81 & 2,11 & 7,04 \\
\hline \multicolumn{10}{|c|}{ Onagraceae } \\
\hline Ludwigia palustris (L.) Elliott & 39 & 6 & 0,19 & 1,26 & 6,50 & 4,11 & 0,25 & 0,66 & 5,02 \\
\hline \multicolumn{10}{|c|}{ Poaceae } \\
\hline Brachiaria subquadripara (Trin.) Hitchc. & 9.938 & 31 & 1,00 & 320,58 & 320,58 & 21,23 & 64,70 & 32,52 & 118,45 \\
\hline Eleusine indica (L.) Gaertn. & 2 & 1 & 0,03 & 0,06 & 2,00 & 0,68 & 0,01 & 0,20 & 0,90 \\
\hline Panicum laxum Sw. & 14 & 3 & 0,10 & 0,45 & 4,67 & 2,05 & 0,09 & 0,47 & 2,62 \\
\hline Paspalum fasciculatum Willd. ex Flüggé & 3.104 & 30 & 0,97 & 100,13 & 103,47 & 20,55 & 20,21 & 10,50 & 51,25 \\
\hline Cynodon dactylon (L.) Pers. & 797 & 2 & 0,06 & 25,71 & 398,50 & 1,37 & 5,19 & 40,43 & 46,99 \\
\hline Setaria parviflora (Poir.) Kerguélen & 75 & 3 & 0,10 & 2,42 & 25,00 & 2,05 & 0,49 & 2,54 & 5,08 \\
\hline \multicolumn{10}{|c|}{ Pontederiaceae } \\
\hline Pontederia rotundifolia L. f. & 2 & 1 & 0,03 & 0,06 & 2,00 & 0,68 & 0,01 & 0,20 & 0,90 \\
\hline \multicolumn{10}{|c|}{ Rubiaceae } \\
\hline Diodia kuntzei (K.) Schum. & 192 & 15 & 0,48 & 6,19 & 12,80 & 10,27 & 1,25 & 1,30 & 12,82 \\
\hline \multicolumn{10}{|c|}{ Linderniaceae } \\
\hline Lindernia crustacea (L.) F. Muell. & 821 & 24 & 0,77 & 26,48 & 34,21 & 16,44 & 5,34 & 3,47 & 25,25 \\
\hline TOTAL & 15.361 & - & 4,70 & 495,50 & 985,70 & 100,00 & 100,00 & 100,00 & 300,00 \\
\hline
\end{tabular}

$\mathrm{NTI}=$ número total de indivíduos; $\mathrm{NPP}=$ número de parcelas presentes; $\mathrm{FRE}=$ frequência; $\mathrm{DEN}=$ densidade; $\mathrm{ABU}=$ abundância; FRR $=$ frequência relativa; $\mathrm{DER}$ = densidade relativa; $\mathrm{ABR}$ = abundância relativa; IVI = índice de valor de importância.

áreas cultivadas com arroz inundado, principalmente para margens de canais (Kissmann $\&$ Groth, 1997). Ainda segundo estes autores, é uma forrageira de excelente aceitação pelo gado, de fácil multiplicação e formação de área verde, porém é considerada tóxica, devido à alta concentração de nitratos nas plantas.

Em estudo realizado por Martins et al. (2003), a B. subquadripara apresentou valores médios de 13,29 t de matéria seca (MS) ha ${ }^{-1}$, $13,26 \mathrm{~g}$ de nitrogênio $\mathrm{kg}^{-1}$ de $\mathrm{MS}$ e relação C/N 3,23.

Atualmente, B. subquadripara é considerada uma ameaça nacional, pois constitui uma grande ameaça à biodiversidade brasileira, uma vez que apresenta densas colonizações em reservatórios de hidrelétricas (Carvalho et al., 2003; Cavenaghi et al., 2003) e em importantes áreas do pantanal (Pott \& Pott, 2004).

P. fasciculatum é uma Poaceae nativa e perene, de ocorrência espontânea nas áreas de várzea da Amazônia Central, onde se destaca devido à sua alta produção de biomassa. $\mathrm{Na}$ região amazônica é conhecida popularmente como "muri" ou "capim mori". O seu ciclo de vida e ecologia demonstram uma estreita adaptação da espécie à instabilidade dos ambientes de várzea (Conserva $\&$ Piedade, 2001). 
Tabela 2 - Famílias e espécies de plantas encontradas na área 2 e respectivos parâmetros fitossociológicos

\begin{tabular}{|c|c|c|c|c|c|c|c|c|c|}
\hline Espécie & NTI & NPP & FRE & DEN & $\mathrm{ABU}$ & FRR & DER & ABR & IVI \\
\hline \multicolumn{10}{|c|}{ Acanthaceae } \\
\hline Justicia pectoralis Jacq. & 1 & 1 & 0,03 & 0,03 & 1,00 & 0,57 & 0,01 & 0,15 & 0,73 \\
\hline \multicolumn{10}{|c|}{ Amarantaceae } \\
\hline Alternanthera philoxeroides (Mart.) Griseb & 76 & 15 & 0,48 & 2,45 & 5,07 & 8,62 & 0,62 & 0,76 & 9,99 \\
\hline \multicolumn{10}{|c|}{ Asteraceae } \\
\hline Ambrosia artemisiifolia $\mathrm{L}$. & 1 & 1 & 0,03 & 0,03 & 1,00 & 0,57 & 0,01 & 0,15 & 0,73 \\
\hline \multicolumn{10}{|c|}{ Cyperaceae } \\
\hline Cyperus compressus L. & 74 & 9 & 0,29 & 2,39 & 8,22 & 5,17 & 0,60 & 1,23 & 7,00 \\
\hline Fimbristylis dichotoma (L.) Vahl & 106 & 3 & 0,10 & 3,42 & 35,33 & 1,72 & 0,86 & 5,29 & 7,87 \\
\hline Fimbristylis miliacea (L.) Vahl & 92 & 8 & 0,26 & 2,97 & 11,50 & 4,60 & 0,75 & 1,72 & 7,06 \\
\hline \multicolumn{10}{|c|}{ Fabaceae - Faboideae } \\
\hline Aeschynomene fluitans Peter & 43 & 14 & 0,45 & 1,39 & 3,07 & 8,05 & 0,35 & 0,46 & 8,85 \\
\hline \multicolumn{10}{|c|}{ Poaceae } \\
\hline Acroceras zizanioides (Kunth) Dandy & 690 & 4 & 0,13 & 22,26 & 172,50 & 2,30 & 5,59 & 25,82 & 33,70 \\
\hline Brachiaria subquadripara (Trin.) Hitchc. & 7.108 & 28 & 0,90 & 229,29 & 253,86 & 16,09 & 57,58 & 37,99 & 111,66 \\
\hline Panicum laxum $\mathrm{Sw}$. & 2 & 2 & 0,06 & 0,06 & 1,00 & 1,15 & 0,02 & 0,15 & 1,32 \\
\hline Paspalum fasciculatum Willd. ex Flüggé & 557 & 24 & 0,77 & 17,97 & 23,21 & 13,79 & 4,51 & 3,47 & 21,78 \\
\hline Cynodon dactylon (L.) Pers. & 2.905 & 24 & 0,77 & 93,71 & 121,04 & 13,79 & 23,53 & 18,11 & 55,44 \\
\hline \multicolumn{10}{|c|}{ Rubiaceae } \\
\hline Diodia kuntzei (K.) Schum. & 170 & 14 & 0,45 & 5,48 & 12,14 & 8,05 & 1,38 & 1,82 & 11,24 \\
\hline \multicolumn{10}{|c|}{ Linderniaceae } \\
\hline Lindernia crustacea (L.) F. Muell. & 520 & 27 & 0,87 & 16,77 & 19,26 & 15,52 & 4,21 & 2,88 & 22,61 \\
\hline TOTAL & 12.345 & - & 5,61 & 398,20 & 668,20 & 100,00 & 100,00 & 100,00 & 300,00 \\
\hline
\end{tabular}

$\mathrm{NTI}=$ número total de indivíduos; $\mathrm{NPP}=$ número de parcelas presentes; FRE = frequência; $\mathrm{DEN}=$ densidade; $\mathrm{ABU}=$ abundância; FRR = frequência relativa; $\mathrm{DER}=$ densidade relativa; $\mathrm{ABR}=$ abundância relativa; $\mathrm{IVI}$ = índice de valor de importância.

Outra espécie que se destacou neste estudo é C. dactylon, que apresenta características nutricionais e produtivas que permitem elevadas produções por animal e por área (Alvim et al., 1999; Vilela et al., 2003), constituindo uma alternativa de forrageira tropical para alimentação de rebanhos leiteiros (Alvim et al., 1997). Essa espécie também se destacou em levantamento fitossociológico, em pastagem de várzea, realizado por Tuffi Santos et al. (2004).

As espécies $A$. fluitans (área 1 e 2) $e$ D. barbatum (área 1), pertencentes à família Fabaceae (subfamília Faboideae), são forrageiras capazes de fixar nitrogênio e constituem-se em alternativa para suprir nitrogênio às pastagens manejadas extensivamente (Thomas, 1992).

O diagrama de Venn e as similaridades florísticas calculadas entre as duas áreas, pelos indices de Jaccard e de Sorensen (Figura 2), mostram que elas são consideradas similares.

Apesar de os indices apresentarem valores distintos ( $\mathrm{Sj}=41$ e IS=58), não houve interferência na interpretação dos resultados, pois, de acordo com Mueller-Dombois \& Ellenberg (1974), são consideradas áreas similares as que apresentam indice de Jaccard maiores que 25 e, no caso do indice de Sorensen, similaridades maiores que 50 são consideradas elevadas. A similaridade entre as duas áreas pode ser decorrente do fato de serem elas vizinhas.

As espécies pertencentes à família Poaceae se destacaram nas pastagens, apresentando maiores valores de frequência, densidade, abundância e IVI. Algumas são consideradas de bom potencial forrageiro, como C. dactylon e o $P$. fasciculatum. No 


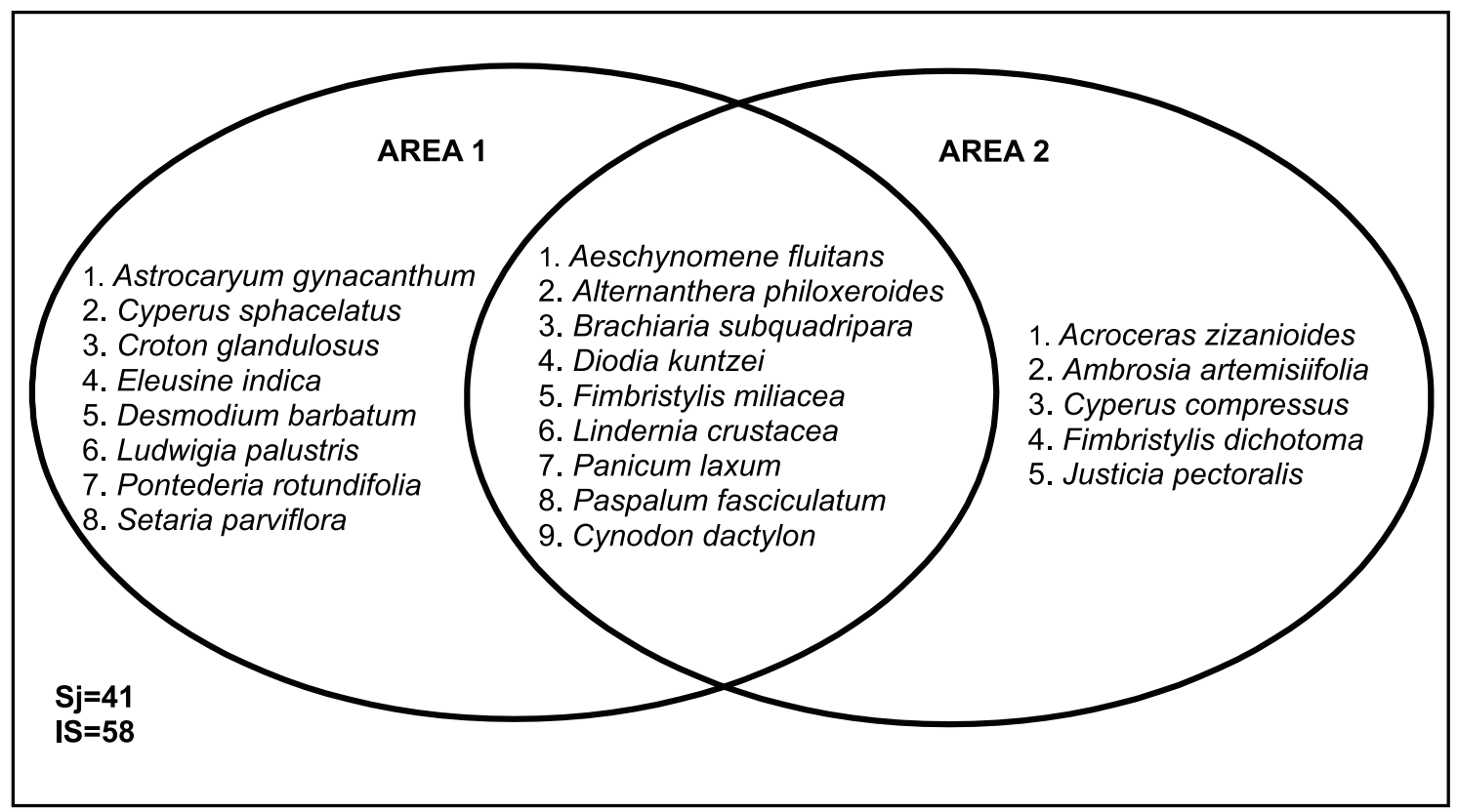

Figura 2 - Diagrama de Venn, ilustrando as espécies exclusivas e compartilhadas entre duas áreas de pastagens de várzea, em Autazes-AM.

entanto, B. subquadripara merece atenção por ser uma planta exótica altamente infestante, que ameaça a biodiversidade brasileira e pode ser tóxica aos animais.

\section{AGRADECIMENTOS}

Aos produtores rurais; ao sr. José Ferreira Ramos, do herbário do INPA; aos amigos do Laboratório de Ciências de Plantas Daninhas da UFAM; e à CAPES/FAPEAM, pela concessão da bolsa à primeira autora e pelo financiamento da pesquisa.

\section{LITERATURA CITADA}

ALBERTINO, S. M. F. et al. Composição florística das plantas daninhas na cultura de guaraná (Paullinia cupana) no Estado do Amazonas. Planta Daninha, v. 22, n. 2, p. $351-358,2004$.

ALBERTINO, S. M. F. et al. Composição florística de plantas daninhas em um lago do rio Solimões, Amazonas Planta Daninha, v. 27, n. 1, p. 1-5, 2009

ALVIM, M. J. et al. Efeito de dois níveis de concentrado sobre a produção de leite de vacas da raça Holandesa em pastagem de "coast-cross" (Cynodon dactylon (L.) Pers.). R. Soc. Bras. Zootec., v. 26, n. 5, p. 967-975, 1997.

Planta Daninha, Viçosa-MG, v. 29, n. 1, p. 69-75, 2011
ALVIM, M. J. et al. Estratégia de fornecimento de concentrado para vacas da raça Holandesa em pastagem de coast-cross. Pesq. Agropec. Bras., v. 34, n. 9, p. 1711-1720, 1999.

CARVALHO, F. T. et al. Plantas aquáticas e nível de infestação das espécies presentes no reservatório de Barra Bonita, no rio Tietê. Planta Daninha, v. 21, p. 15-19, 2003. (Edição Especial)

CAVENAGHI, A. L. et al. Caracterização da qualidade da água e sedimento relacionados com a ocorrência de plantas aquáticas em cinco reservatórios da bacia do rio Tietê. Planta Daninha, v. 21, p. 43-52, 2003. (Edição Especial)

CONSERVA, A. S.; PIEDADE, M. T. F. Ciclo de vida e ecologia de Paspalum fasciculatum Willd. Ex. Fluegge (Poaceae), na Várzea da Amazônia Central. Acta Amaz., v. 31, n. 2, p. 205-220, 2001.

COSTA, C. et al. Evolução das pastagens cultivadas e do efetivo bovino no Brasil. Veter. Zootec., v. 15, n. 1, p. 8-17, 2008.

INSTITUTO BRASILEIRO DE GEOGRAFIA E ESTATÍSTICA - IBGE. Pesquisa pecuária municipal. Disponível em: http://www.sidra.ibge.gov.br. Acesso em: 27 de jan. de 2010 .

KISSMANN, K. G.; GROTH, D. Plantas infestantes e nocivas. 2.ed. São Paulo: BASF, 1997. v. 1.824 p. 
LARA, J. F. R.; MACEDO, J. F.; BRANDÃO, M. Plantas daninhas em pastagens de várzeas do estado de Minas Gerais. Planta Daninha, v. 21, n. 1, p. 11-20, 2003.

MACIEL, C. D. G. et al. Composição florística da comunidade infestante em gramados de Paspalum notatum no município de Assis, SP. Planta Daninha, v. 26, n. 1, p. 5764, 2008.

MARTINS, D. et al. Caracterização da comunidade de plantas aquáticas de dezoito reservatórios pertencentes a cinco bacias hidrográficas do Estado de São Paulo. Planta Daninha, v. 26, n. 1, p. $17-32,2008$

MARTINS, D. et al. Caracterização química das plantas aquáticas coletadas no reservatório de Salto Grande (Americana-SP). Planta Daninha, v. 21, n. 1, p. 21-25, 2003 (Edição Especial)

MASCARENHAS, M. H. T. et al. Flora infestante em pastagem degradada sob recuperação, pelo sistema de integração lavoura-pecuária, em região de cerrado. R. Bras. Milho Sorgo, v. 8, n. 1, p. 41-55, 2009.

MODESTO JÚNIOR, M. S.; MASCARENHAS, R. E. B. Levantamento da infestação de plantas daninhas associada a uma pastagem cultivada de baixa produtividade no nordeste paraense. Planta Daninha, v. 19, n. 1, p. 11-21, 2001.

MUELLER-DOMBOIS, D.; ELLENBERG, H. Aims and methods of vegetation ecology. New York: John Wiley \& Sons, 1974. 547 p.

OHLY, J.; HUND, M. Pasture farming on the floodplains of Central Amazonia. Animal Res. Develop., n. 43/44, p. 53-79, 1996.
OLIVEIRA, A. N.; AMARAL, I. L. Florística e fitossociologia de uma floresta de vertente na Amazônia Central, Amazonas, Brasil. Acta Amaz., v. 34, n. 1, p. 21-34, 2004.

PEIXOTO, A. L.; CARVALHO, S. M.; DA ROSA, M. M. T. Análise botânica de um campo de pastagem no Estado do Rio de Janeiro. Planta Daninha, v. 5, n. 2, p. 1-7, 1982.

POTT, A.; POTT, V. J. Features and conservation of the Brazilian Pantanal wetland. Wetlands Ecol. Manag., v. 12, p. $547-552,2004$.

SOUZA, L. S. A.; SILVA, J. F.; SOUZA, M. D. B. Composição florística de plantas daninhas em agrossistemas de cupuaçuzeiro (Theobroma grandiflorum) e pupunheira (Bactris gasipaes). Planta Daninha, v. 21, n. 2, p. 249-255, 2003.

THOMAS, R. J. The role of legume in the nitrogen cycle of productive and sustainable pastures. Grass Forage Sci., v. 47, n. 2, p. 133-142, 1992.

THOMAZ, S. M. et al. Temporal trends and effects of diversity on occurrence of exotic macrophytes in a large reservoir. Acta Oecol., v. 35, p. 614-620, 2009.

TUFFI SANTOS, L. D. et al. Levantamento fitossociológico em pastagens degradadas sob condições de várzea.

Planta Daninha, v. 22, n. 3, p. 343-349, 2004.

VILELA, D. et al. Utilização de soja integral tostada na dieta de vacas em lactação, em pastagem de coast-cross (Cynodon dactylon L. Pers.). R. Bras. Zootec., v. 32, n. 5, p. 1243-1249, 2003. 